\title{
Slip Flow in a Magnetohydrodynamic Boundary Layer
}

\author{
Michael James Martin, ${ }^{1}$ Chunpei Cai, ${ }^{2}$ and Iain D. Boyd ${ }^{3}$ \\ ${ }^{1}$ Department of Mechanical Engineering, Louisiana State University, Baton Rouge, LA. Senior Member, AIAA \\ ${ }^{2}$ Department of Mechanical and Aerospace Engineering, New Mexico State University, Las Cruces, NM. Senior \\ Member, AIAA \\ ${ }^{3}$ Department of Aerospace Engineering, University of Michigan, Ann Arbor, MI. Fellow, AIAA
}

The flow in a laminar boundary layer of an electrically conducting gas near the continuum limit, with a slip boundary condition at the wall, is computed numerically. Both the magnetic terms and the slip boundary condition will lead to a loss of self-similarity in the flow. The structure of the equations shows that the importance of the magnetic terms increases as the flow becomes more rarefied. Numerical results provide an estimate of the relative impact of the MHD terms on the wall friction and slip velocity in a rarefied boundary layer. The results suggest that the high gradients created by MHD terms will lead to continuum breakdown, increasing the importance of the slip condition.

\section{Nomemclature}

$\begin{array}{lll}\mathrm{A}_{1} & = & \text { Combined electric and magnetic field scaling constant } \\ \mathrm{A}_{2} & = & \text { Magnetic field scaling constant } \\ \mathrm{B}_{\mathrm{o}} & = & \text { Magnetic field strength } \\ \mathrm{E}_{\mathrm{Z}} & = & \text { Electric field strengh } \\ f & = & \text { Non-dimensional stream function } \\ \mathrm{L} & = & \text { Length } \\ \mathrm{K} & = & \text { Non-dimensional co-ordinate } \\ \mathrm{Kn} & = & \text { Knudsen number } \\ \mathrm{Re} & = & \text { Reynolds number } \\ \mathrm{u} & = & \text { Velocity in the x-direction } \\ \mathrm{v} & = & \text { Velocity in the y-direction } \\ \mathrm{x} & = & \text { Co-ordinate tangential along the plate } \\ \mathrm{y} & = & \text { Co-ordinate normal to the plate } \\ \eta & = & \text { Non-dimensional co-ordinate } \\ \lambda & = & \text { Mean free path } \\ \mu & = & \text { Dynamic viscosity } \\ v & = & \text { Kinematic viscosity } \\ \rho & = & \text { Density } \\ \sigma & = & \text { Electrical conductivity } \\ \sigma_{\mathrm{m}} & = & \text { Tangential momentum accommodation coefficient } \\ \psi & = & \text { Stream function } \\ \mathrm{Subscript} & & \\ \mathrm{g} & = & \text { Gas } \\ \mathrm{o} & = & \text { Free-stream } \\ \mathrm{s} & = & \text { Slip } \\ \mathrm{w} & = & \text { Wall }\end{array}$

\section{Introduction}

The laminar boundary layer over a flat plate is one of the fundamental problems in fluid dynamics. The classic Blasius solution solves this problem through a combination of dimensional scaling and numerical solution [1-2]. Previous researchers have studied the impact of adding magneto-hydrodynamic terms to the problem, but were only able to obtain solutions to the Blasius boundary layer when the field strengths scaled with the square root of distance along the plate [3]. 
The boundary layer in slip flow has also been studied. Initial work suggested that there would be rarefied flow effects as the boundary layer Knudsen number approached 0.001 [4]. In these cases, the velocity slip [5] and temperature jump [6] boundary conditions would become appropriate. Initial results based on perturbation analysis suggested that these effects would be negligible [7]. However, more complete numerical analysis, which incorporated the loss of self-similarity, showed the slip and temperature jump boundary conditions led to changes in the wall friction, boundary layer thickness, and heat transfer for the Blasius boundary layer [8] and the related Falkner-Skan boundary layer [9].

In magneto-hydrodynamic power generation, the flow may be rarefied as well [10]. Only one previous attempt has been made to combine magneto-hydrodynamic terms with slip terms in a boundary layer solution [11]. In that case, the fields were all assumed to scale with the square root of distance along the plate, and the plate was assumed to be stretching in a way where the slip boundary condition did not cause a loss of self-similarity.

The present work combines the magneto-hydrodynamic terms with velocity slip and temperature jump boundary conditions in a way that accounts for the loss of self-similarity in the solution. The work begins with formulation of the non-dimensional governing equations of slip flow with MHD terms. Numerical results are presented for a range of magnetic and electrical fields. Based on these results, qualitative assesments of how slip and MHD terms interact are provided.

\section{III.Formulation}

The problem geometry is shown in Fig. (1). The gas flows over a plate with a free-stream velocity $\mathrm{u}_{\mathrm{o}}$. As flow moves along the plate direction $\mathrm{x}$, a boundary layer grows along the plate, with a thickness proportional to the square root of $\mathrm{x}$. All gradients in the $\mathrm{x}$-direction are assumed to be smaller than the gradients in the $\mathrm{y}$-direction, and the external free-stream velocity and pressure are constant. There is a uniform magnetic field with a strength $B_{0}$ acting in the y-direction. The electric field acts in the z-direction with a strength $\mathrm{E}_{\mathrm{z}}$. This condition is similar to the classical analysis [3], without the requirement that the fields be matched to the boundary layer thickness.

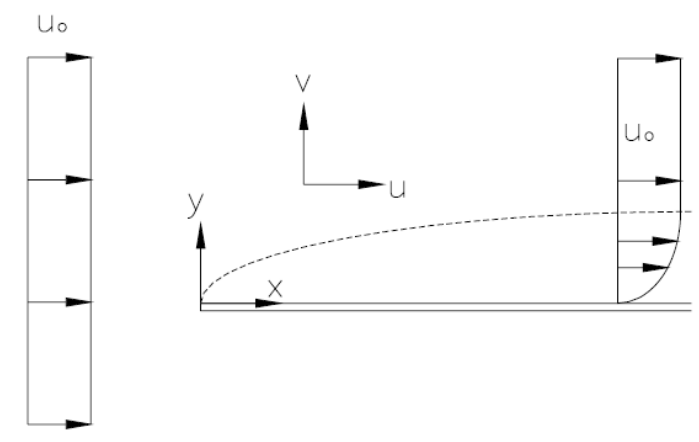

Fig. 1. Boundary layer flow over a flat plate.

The x-momentum equation for the laminar boundary layer over a flat plate with no pressure gradient, including and magneto-hydrodynamic terms, is given as:

$$
u \frac{\partial u}{\partial x}+v \frac{\partial u}{\partial y}=v \frac{\partial^{2} u}{\partial y^{2}}-\frac{\sigma \cdot B_{o} \cdot E_{z}}{\rho}-\frac{\sigma \cdot B_{o}^{2}}{\rho} u
$$

where $\mathrm{u}$ and $\mathrm{v}$ are the velocities in the $\mathrm{x}$ - and $\mathrm{y}$ - directions, $\mathrm{x}$ is the direction along the plate, $\mathrm{y}$ is the direction normal to the plate, $v$ is the kinematic viscosity, $\sigma$ is the electrical conductivity, $\rho$ is the density, $E_{z}$ is the electrical field strength, and $\mathrm{B}_{\mathrm{o}}$ is the magnetic field strength. The left-hand side of this equation represents fluid advection, while the right-hand side combines viscous stresses, the $\mathrm{E} \times \mathrm{B}$ term, and the magnetic pressure.

The velocities $\mathrm{u}$ and $\mathrm{v}$ will be functions of the stream function $\Psi$ : 


$$
\begin{gathered}
u=\frac{\partial \Psi(x, y)}{\partial y} \\
v=-\frac{\partial \Psi(x, y)}{\partial x}
\end{gathered}
$$

These equations can then be transformed, using the non-dimensionalizations and non-dimensional stream functions given below:

$$
\begin{gathered}
x^{*}=x / L \\
y^{*}=\frac{y}{\left(v \mathrm{~L} / \mathrm{u}_{0}\right)^{1 / 2}} \\
u^{*}=u / u_{o} \\
v^{*}=\frac{v}{\left(v u_{o} / x\right)^{1 / 2}} \\
y^{*}=\frac{y /\left(v \mathrm{~L} / \mathrm{u}_{0}\right)^{1 / 2}}{\left(x^{*}\right)^{1 / 2}}=\frac{y}{\left.(v / \mathrm{L})^{1 / 2} / \mathrm{u}_{0}\right)^{1 / 2}} \\
u^{*}=\frac{u}{u_{o}}=f^{\prime}(\eta) \\
v^{*}=\frac{v}{\left(v u_{0} / x\right)^{1 / 2}}=0.5\left[\eta f^{\prime}(\eta)-f(\eta)\right]
\end{gathered}
$$

where $\mathrm{L}$ is the length of the flat plate.

When the flow becomes rarefied, the no-slip condition is replaced by the slip-flow condition:

$$
u_{\text {slip }}=\mathrm{u}_{\mathrm{g}}-\mathrm{u}_{\mathrm{w}}=\left.\lambda \cdot \frac{\left(2-\sigma_{m}\right)}{\sigma_{m}} \frac{\partial \mathrm{u}}{\partial n}\right|_{\text {wall }}+\left.\frac{3}{4} \cdot \frac{\mu}{\rho \cdot T_{g}} \frac{\partial \mathrm{T}}{\partial s}\right|_{\text {wall }}
$$

where $u_{\text {slip }}$ is the wall slip velocity, $u_{g}$ is the gas velocity at the wall, $u_{w}$ is the wall velocity, $\sigma_{m}$ is the tangential momentum accommodation coefficient, $\mathrm{n}$ is the wall normal direction, $\mu$ is the viscosity, $\mathrm{T}_{\mathrm{g}}$ is the gas temperature, and $\mathrm{s}$ is the wall tangential direction.

If the wall is isothermal, the non-dimensionalizations given in (4) through (10) can be used to nondimensionalize the slip boundary condition to obtain

$$
f^{\prime}(0)=\frac{\left(2-\sigma_{m}\right)}{\sigma_{m}} \mathrm{Kn}_{\mathrm{x}} \operatorname{Re}_{x}^{1 / 2} f^{\prime \prime}(0)=\mathrm{K} f^{\prime \prime}(0)
$$

where $\mathrm{Kn}_{\mathrm{x}}$ and $\mathrm{Re}_{\mathrm{x}}$ are the Knudsen and Reynolds numbers based on $\mathrm{x}$, and $\mathrm{K}$ is a non-dimensional parameter that describes the behavior at the surface: 


$$
\mathrm{K}=\frac{\left(2-\sigma_{m}\right)}{\sigma_{m}} \mathrm{Kn}_{\mathrm{x}} \operatorname{Re}_{x}^{1 / 2}
$$

When these non-dimensionalizations are applied to equation (1), the following partial differential equation is obtained:

$$
\frac{\partial^{3} f}{\partial \eta^{3}}=-0.5 f \frac{\partial^{2} f}{\partial \eta^{2}}-0.5 K \frac{\partial}{\partial \eta}\left[\frac{\partial f}{\partial \eta} \frac{\partial f}{\partial K}\right]+\frac{\mathrm{A}_{1}}{\mathrm{~K}^{2}}+\frac{\mathrm{A}_{2}}{\mathrm{~K}^{2}} \frac{\partial f}{\partial \eta}
$$

where $\mathrm{A}_{1}$ and $\mathrm{A}_{2}$ are non-dimensionalized MHD terms:

$$
\begin{gathered}
\mathrm{A}_{1}=\frac{\sigma \cdot B_{o} \cdot E_{z}}{\mu}\left[\frac{\left(2-\sigma_{m}\right)}{\sigma_{m}} \lambda\right]^{2}=\left(\frac{E_{z}}{B_{o}}\right) \cdot \mathrm{A}_{2} \\
\mathrm{~A}_{2}=\frac{\sigma \cdot B_{o}^{2}}{\mu}\left[\frac{\left(2-\sigma_{m}\right)}{\sigma_{m}} \lambda\right]^{2} .
\end{gathered}
$$

This scaling indicates that if $A_{2}$ is equal to zero, than $A_{1}$ must also be equal to zero. This restricts the parameter space of the problem.

These equations can be solved with a marching scheme, beginning from a uniform flow at large values of $\mathrm{K}_{1}$, and moving to smaller values of $K_{1}$, as done for the slip boundary layer in earlier work [8-9]. Because $K_{1}$ is inversely proportional to the square root of $\mathrm{x}$, this corresponds to marching from the leading edge of the plate, similar to what is done with no-slip boundary layers in dimensional solutions [10].

\section{IV.Results}

Two sets of cases are considered. In the first set of cases, $A_{1}$ is set to zero, and $A_{2}$ is set to $0,0.02,0.04,0.06$, 0.08 , and 0.1. In the second set of cases, $A_{2}$ is set to 0.02 , and $A_{1}$ is set to $0,0.02,0.04,0.06,0.08$, and 0.1 .. Figures (2) and (3) show the non-dimensional wall shear stress $f$ " $(0)$ and the non-dimensional wall velocity for the first set of cases. Figures (4) and (5) show the non-dimensional wall shear stress $f$ " $(0)$ and the non-dimensional wall velocity for the second set of cases.

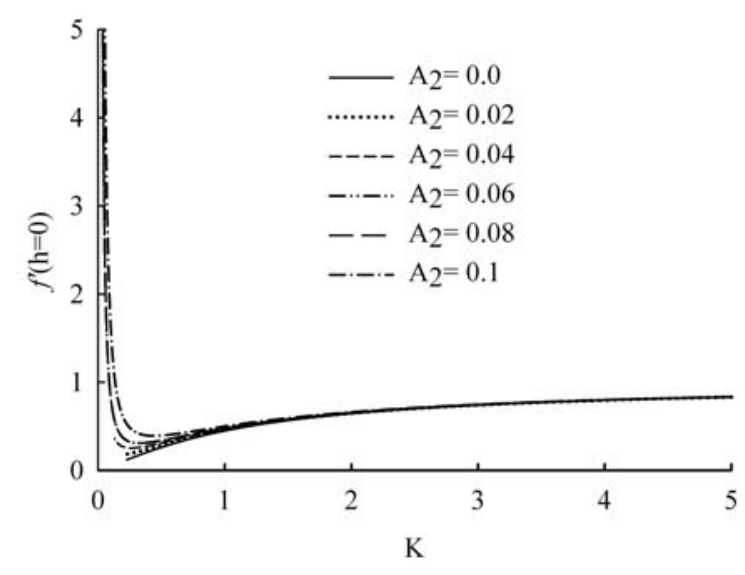

Fig. 2. $f^{\prime}(0)$ vs $K$ for $A_{1}=0, A_{2}=0.0,0.02,0.04,0.06,0.08$, and 0.1 


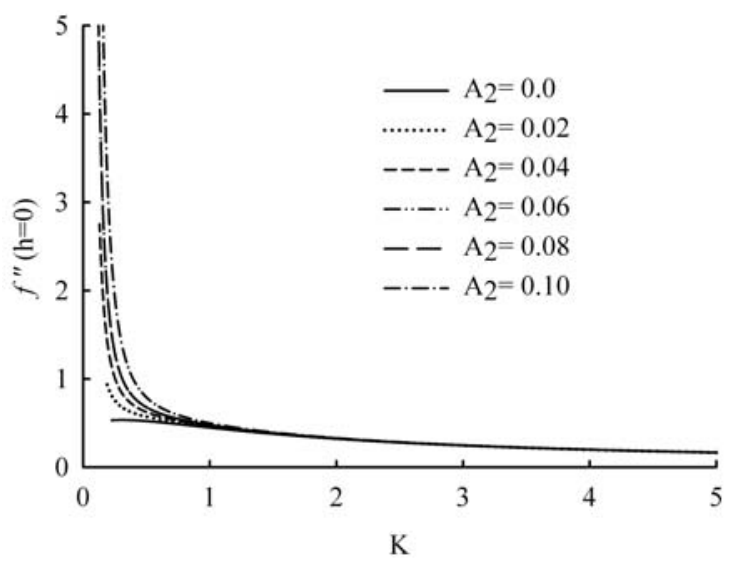

Fig. 3. $f$ "(0) vs $K$ for $A_{1}=0, A_{2}=0.0,0.02,0.04,0.06,0.08$, and 0.1

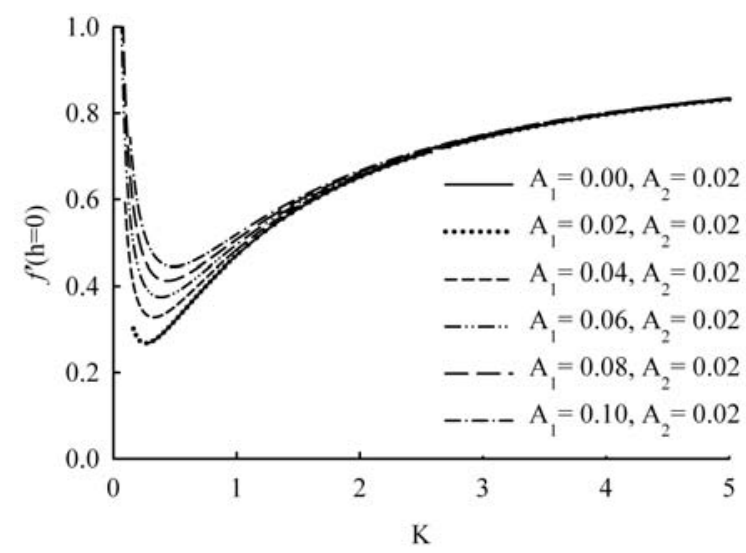

Fig. 4. $f^{\prime}(0)$ vs $K$ for $A_{2}=0.02, A_{1}=0.0,0.02,0.04,0.06,0.08$, and 0.1

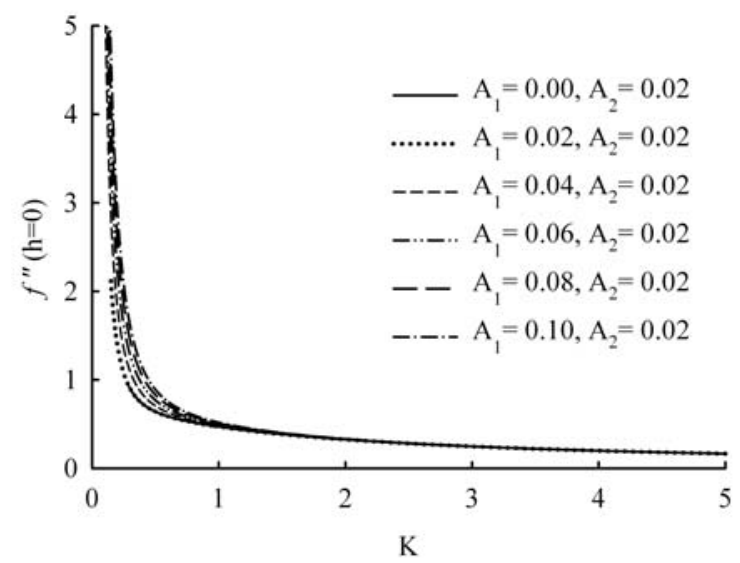

Fig. 5. $f "(0)$ vs $\mathrm{K}$ for $A_{2}=0.02, A_{1}=0.0,0.02,0.04,0.06,0.08$, and 0.1

These results show that when $\mathrm{K}_{1}$ is less than zero, and the non-dimensional field strengths are on the order of magnitude of 0.01 , there is an increase in the wall shear stress and slip velocity due to the MHD effects. This suggests that the rarefied flow effects.

The effect of the magneto-hydrodynamic terms on the flow can be understood by looking at the velocity profiles. Figure 6 shows the non-dimensional velocity profiles at $\mathrm{K}=0.3$ when both $\mathrm{A}_{1}$ is equal to zero, and $\mathrm{A}_{2}$ varies from zero to 0.10 . are equal to zero. The magnetic pressure terms create a velocity bulge in the boundary 
layer, identical to the result without the slip condition. The slip velocity at the wall in the MHD cases is much larger than in the non-MHD cases due to the increased gradient. This shows that the interaction between wall nonequilibrium and MHD terms is significant and must be accounted for in computations. The results also show that because of the unusual shape of the MHD boundary layer, the boundary layer thickness many no longer be the approporate scaling for the effective Knudsen number

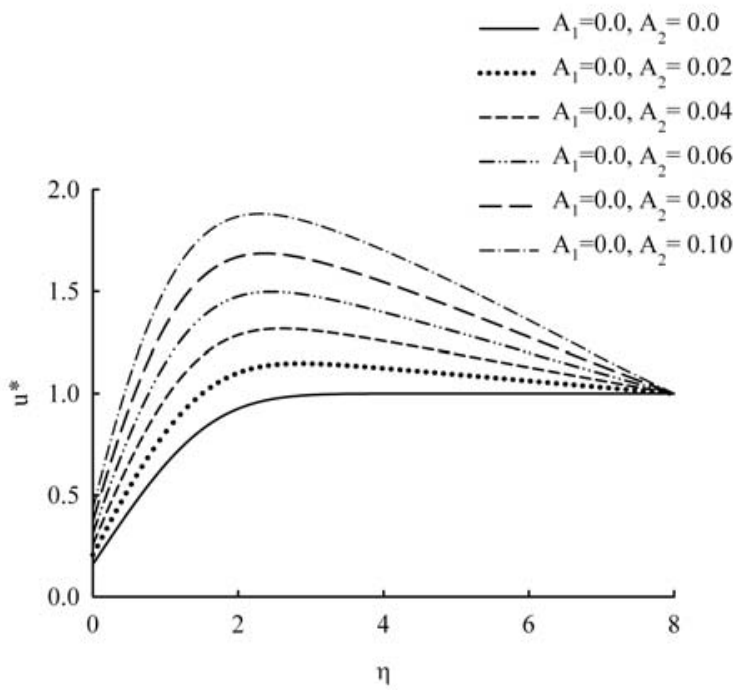

Fig 6.a. $u^{*}$ vs $\eta$

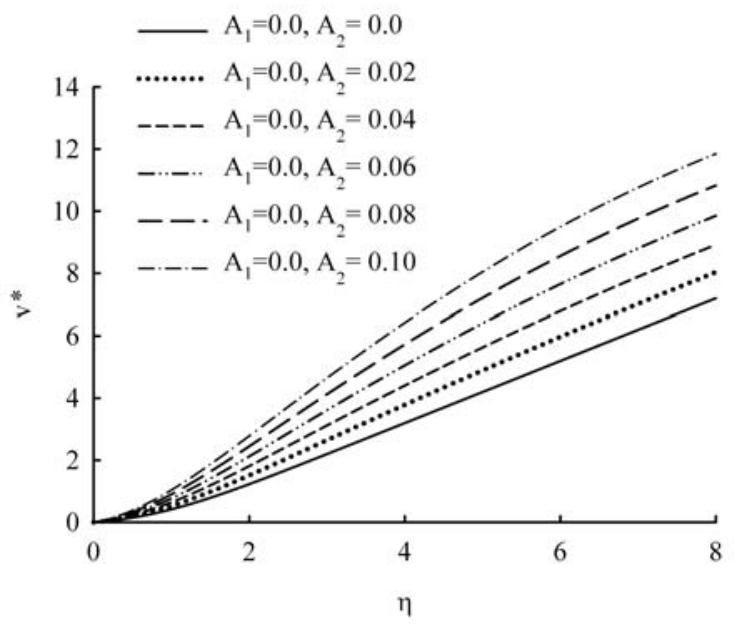

Fig 6.b. $\mathbf{v}^{*}$ vs $\eta$

Fig. 6. Velocity Profiles for $A_{1}=0, A_{2}=0,0.02,0.04,0.06,0.08$, and 0.10

Figures 7 ashows the non-dimensional velocity profiles at $\mathrm{K}=0.3$ when $\mathrm{A}_{1}$ varies from 0 to 0.10 , and $\mathrm{A}_{2}$ is equal to 0.02 . The $\mathrm{E} \times \mathrm{B}$ ters further increase the bulge in the velocity profile, increasing the significance of wall non-equilibrium.

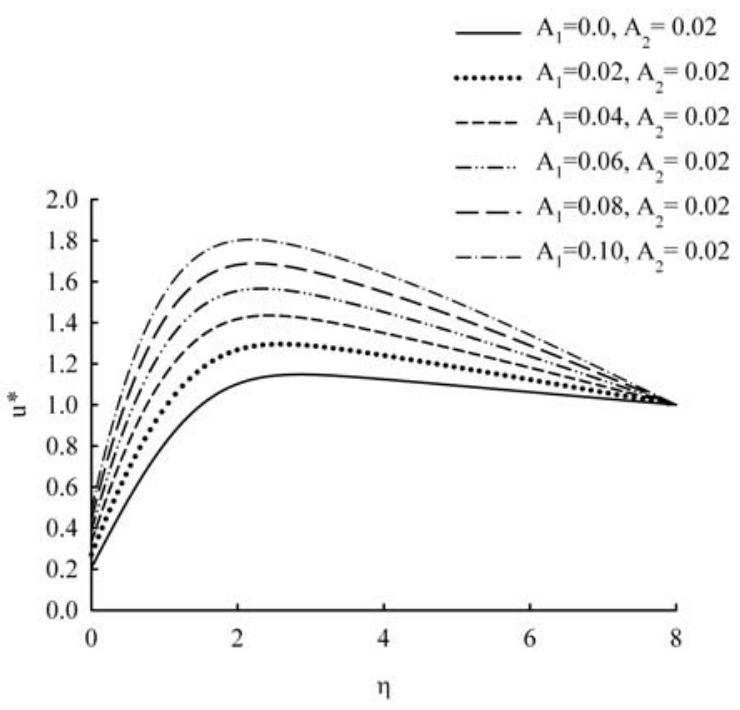

Fig 7.a. $\mathbf{u}^{*}$ vs $\eta$

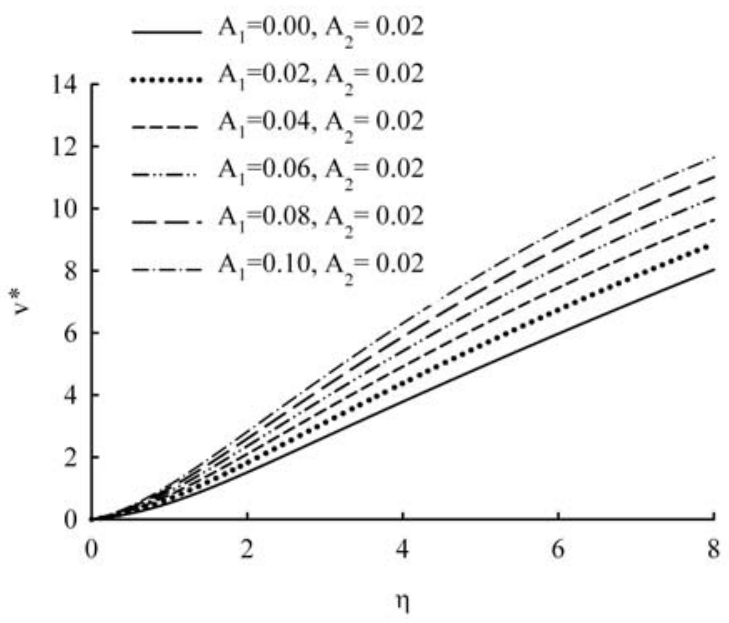

Fig 7.b. $v^{*}$ vs $\eta$

Fig. 7. Velocity Profiles for $A_{1}=0,0.02,0.04,0.06,0.08$, and $0.01, A_{2}=0.02$ 


\section{Conclusions}

This work shows that a scaling can be created that combines the effects of non-equilibrium gas-surface interactions and magneto-hydrodynamic terms in the boundary layer equations. The work shows that because of the high gradients created by MHD terms, the effect of a slip boundary condition is magnified compared to non-MHD flows. In all cases, the self-similiarity of the boundary layer is lost, unless the fields and the slip both are made to scale with the boundary layer growth.

\section{Acknowledgements}

The authors wish to thank the Louisiana Optical Network Initiative for computational resources. The lead author would also like to thank Elham Maghsoudi for her help in preparation of the final plots for this paper.

\section{References}

[1] Blasius, H. "Grezzschichten in Flüssigkeiten mit kleiner Reibung”, Zeitschrift für Mathematik und Physik, Vol. 56, No. 1, 1908, pp. 1-37.

[2] Schlichting, H., and Gersten, K. Boundary Layer Theory, McGraw-Hill, New York, 2000.

[3] Sutton, G. W., and Sherman, A. Engineering Magnetohydrodynamics, McGraw-Hill, New York, 1965.

[4] Kogan, M. N. Rarefied Gas Dynamics, Plenum Press, New York, 1969.

[5] Maxwell, J. C. "On Stresses in Rarefied Gases Arising from Inequalities of Temperature," Philosophical Transactions of the Royal Society of London, Vol. 170, 1879, pp. 231-256.

[6] Smoluchowski von Smolan, M. "Über Wärmeleitung in verdünnten Gasen," Annalen der Physik und Chemi, Vol. 64 , No. 1, 1898, pp. 101-130

[7] Maslen, S. H., "Second Approximation to laminar compressible boundary layer on flat plate in slip flow," NACA TN 2818, 1952.

[8] Martin, M. J., and Boyd, I. D., "Momentum and Heat Transfer in a laminar boundary layer with slip flow," AIAA Journal of Thermophysics and Heat Transfer, Vol. 20, No. 4, 2006, pp. 710-719.

[9] Martin, M. J., and Boyd, I. D., "Falkner-Skan Flow over a Wedge with Slip Boundary Conditions," AIAA Journal of Thermophysics and Heat Transfer, Vol. 24, No. 2, 2010, pp. 263-270.

[10] Andersson, H. I., and De Korte, E., "MHD flow of a power-law fluid over a rotating disk," Journal of Mechanics, Vol. 21, No. 3, 2002, pp. 317-324.

[11] Bhattacharyya, K., Mukhopadhyay, S., and Layek, G. C., " MHD Boundary Layer Slip Flow and Heat Transfer over a Flat Plate," Chinese Physics Letters, Vol. 28, No. 2, 2011, pp. Article 024701. 